\title{
Evaluation of Surface Modification as a Lunar Dust Mitigation Strategy for Thermal Control Surfaces
}

\author{
James R. Gaier ${ }^{1}$, Deborah L. Waters ${ }^{2}$, and Robert M. Misconin ${ }^{3}$ \\ NASA Glenn Research Center, Cleveland, OH 44135 \\ Bruce A. Banks ${ }^{4}$ \\ Alphaport, Cleveland, $\mathrm{OH} 44135$ \\ Mark Crowder ${ }^{5}$ \\ Ball Aerospace, Boulder, CO 80301
}

\begin{abstract}
Three surface treatments were evaluated for their ability to lower the adhesion between lunar simulant dust and AZ93, AIFEP, and AgFEP thermal control surfaces under simulated lunar conditions. Samples were dusted in situ and exposed to a standardized puff of nitrogen gas. Thermal performance before dusting, after dusting, and after part of the dust was removed by the puff of gas, were compared to perform the assessment. None of the surface treatments was found to significantly affect the adhesion of lunar simulants to AZ93 thermal control paint. Oxygen ion beam texturing also did not lower the adhesion of lunar simulant dust to AIFEP or AgFEP. But a workfunction matching coating and a proprietary Ball Aerospace surface treatment were both found to significantly lower the adhesion of lunar simulants to AIFEP and AgFEP. Based on these results, it is recommended that all these two techniques be further explored as dust mitigation coatings for AIFEP and AgFEP thermal control surfaces.
\end{abstract}

\section{Nomenclature}

AgFEP $=0.24 \mathrm{~mm}(0.010$ in.) thick fluorinated ethylene propylene (FEP) with a silver reflecting surface on the back

AlFEP $=0.13 \mathrm{~mm}(0.005 \mathrm{in}$.) thick FEP with an aluminum reflecting surface on the back

$\mathrm{AxFEP}=$ both AgFEP and AlFEP

AZ93 = a white thermal control paint formulated by AZ Technologies similar to Z93

$\alpha=$ absorptivity over the solar spectrum $(250$ to $2500 \mathrm{~nm})$

$\alpha_{\mathrm{rel}} \quad=\alpha / \alpha$ of pristine surface

$\varepsilon=$ emissivity over thermal range ( 100 to $400 \mathrm{~K})$

$\varepsilon_{\text {rel }} \quad=\varepsilon / \varepsilon$ of pristine surface

\section{Introduction}

$\mathrm{D}$ URING the Apollo program, lunar surface operations were hampered by the effects of a fine, pervasive, highly adhesive dust. The mission records contain references to challenges involving obscuration of vision, clogging of equipment, coating of surfaces, abrasion of surfaces, degradation of seal performance, degradation of thermal performance, and minor health issues. ${ }^{1}$ Some of the potentially most serious consequences were due to lunar dust on thermal control surfaces, which caused overheating in several of the science experiments and the batteries of the lunar roving vehicle (LRV). ${ }^{2}$ Recent studies using lunar simulant dusts sprinkled onto thermal control surface samples in a simulated lunar environment suggest that, depending on the nature of the dust, the degradation of

\footnotetext{
${ }^{1}$ Research Physicist, Space Environment and Experiments Branch, 21000 Brookpark Road, and Member AIAA.

${ }^{2}$ Materials Engineer, Space Environment and Experiments Branch, 21000 Brookpark Road.

${ }^{3}$ LERCIP Summer Intern, Space Environment and Experiments Branch, 21000 Brookpark Road.

${ }^{4}$ Senior Physicist, Space Environment and Experiments Branch, 21000 Brookpark Road.

${ }^{5}$ Staff Consultant, Ball Aerospace, 1600 Commerce St., Boulder, and Member AIAA.
} 
performance, as measured by the ratio of the solar absorptance $(\alpha)$ to the thermal emittance $(\varepsilon)$, will be substantial, perhaps by as much as a factor of 3.5 . $^{3}$ Increasing the thermal control surface area by such a factor is not a realistic option. It seems clear that before extensive lunar exploration efforts can continue, strategies must be developed to mitigate these effects.

A wide variety of approaches have been suggested to mitigate the effects of dust. There are three principal approaches. The thermal control surface can somehow be made more dust tolerant, technology can be developed to decrease the chances of dust attaching to the surface, or technology can be developed to remove the dust from the surfaces. The technology development has generally followed one of two philosophies, active removal of the dust or prevention of its accumulation, and passive surfaces that keep the dust from adhering to the surfaces.

Passive dust mitigation surfaces have the distinct advantage of not requiring the input of energy. This is desirable both because spacecraft and surface systems are often energy limited, and because there is the possibility of electrical or mechanical failure in an active system. Passive dust mitigation technologies work to control charge transfer, in an effort to minimize the electrostatic forces which are key to adhesion, and to decrease the adhesion forces between the surface and the dust. The adhesion between the dust and the thermal control surface can be lessened by changing the surface chemistry and the surface texture.

The simplest method to decrease the dust adhesion is by adding texture without changing the surface chemistry. By keeping the surface chemistry of the pristine surface, it is expected that the thermal optical properties of $\alpha$ and $\varepsilon$ will be similar to that of the pristine surface as well. The texture that is desired is that of closely spaced cones, in essence a bed of microscopic nails. The thought is that each dust particle will be supported by only a few of these cones, drastically reducing its contact area. The cone size and spacing are critically important. If they are too widely spaced the dust particles can be trapped between the cones which may in fact lead to an increase in the contact area, and so an increase in adhesion. If the cones are too narrowly spaced, then the contact area will not differ much from the pristine surface, and there will be little advantage to the texture. Dust particles are generally described by the lunar dust community as those being smaller than $20 \mu \mathrm{m}$. Recent studies have shown that a significant number of lunar regolith particles are as small as $0.05 \mu \mathrm{m} .{ }^{4,5}$ So dust particles on the Moon range in size over a factor of 400 . It would be difficult to design a textured surface that would work for all. But the JSC-1AF test dust had few submicron particles and so the size ranged over only about a factor of 20 .

A preliminary proof-of-concept study was undertaken to determine whether the strategy of texturing the thermal control surfaces is a promising technology to pursue. Three thermal control surfaces, a white paint (AZ-93) and two second surface mirrors (AgFEP and AlFEP) were textured at two to four levels using an oxygen ion beam to etch away part of the surfaces leaving a cone structure. The size and spacing of the cones depended upon the time exposed to the ion beam. Since this is a passive technique and the dust was gently sifted onto the samples from above, relative dust adhesion was determined by exposing each dusted sample to a standard puff of nitrogen gas. The intent was not to demonstrate that the standard puff would totally clean the surfaces, but to compare the extent of dust removal to an untreated surface.

Of the adhesion forces present at the lunar surface, only electrostatic forces have the capability of attracting dust particles to spacecraft surfaces from a distance. Electrostatic forces have been shown to be important in cohesion and adhesion of lunar dust particles. ${ }^{6}$ Although there are multiple charging mechanisms at work in the lunar environment, ${ }^{7}$ tribo-charging will probably be the most important anthropogenic charging mechanism. During tribocharging electrons are transferred from a material that easily loses electron (i.e., has a low work function) to a material that holds tightly onto its electron (i.e., has a high work function). So tribo-charging is minimized if the work function of the two surfaces is similar. The approach to a dust resistant coating evaluated here is to apply a coating to the thermal control surface that has a work function that matches the dust as closely as possible.

Although there have been studies that estimate the work function of the lunar dust, ${ }^{8}$ perhaps the best match would be a coating made from the dust itself. So a sputter target was made from a slurry of the lunar stimulant NU-LHT-1D. This was used to coat AZ-93, AgFEP, and AlFEP samples with a coating a few tens of nm thick using a dual ion beam sputter deposition system.

A proof-of-concept study was undertaken to determine whether the strategy of matching the work function of thermal control surfaces to the dust is a promising technology to pursue. Three thermal control surfaces, a white paint (AZ-93) and two second surface mirrors (AgFEP and AlFEP) were coated at two levels with the work function matching coating. The same standard puff of nitrogen test that was used with the textured surfaces was also used to determine the effectiveness of these surfaces. 
A proprietary surface modification process ${ }^{9}$ developed by Ball Aerospace was also included in this evaluation. An ensemble of treated surfaces was studied through a Lunar Advanced Science and Exploration Research (LASER) grant ${ }^{10}$ to verify the viability of particle adhesion reduction to space environments. In this work the Ball process was applied to applied to both AZ93 and AgFEP samples. In addition to testing the Ball surface treatment using the nitrogen puff method, four AZ93 and four AgFEP samples was tested using advanced brushing techniques.

\section{Methods and Materials}

Three types of thermal control surfaces were applied to $2.54 \mathrm{~cm}(1.00 \mathrm{in}$.) diameter, $0.64 \mathrm{~cm}(0.25 \mathrm{in}$.) thick aluminum substrates. The surfaces included aluminum-backed $130 \mu \mathrm{m}(0.005$ in. $)$ thick FEP, $250 \mu \mathrm{m}(0.010 \mathrm{in}$. $)$ thick FEP backed with silver and an inconel oxidation protection layer (both from Sheldahl), and AZ93 thermal control paint (AZ Technologies). In order to minimize heat losses, the substrates were suspended from the edges in the sample holder by two layers of $250 \mu \mathrm{m}$ thick layers of Kapton ${ }^{\circledR}$, and temperature was measured using $130 \mu \mathrm{m}$ diameter(AWG 36) type K calibrated thermocouples affixed to the back of each sample.

The AZ93 and AxFEP (meaning either AlFEP or AgFEP) surfaces were textures using hyperthermal energy oxygen ions in the Atomic Oxygen Exposure Facility at the NASA Glenn Research Center. The exposure was performed at normal incidence using a Veeco Mark II end Hall ion source, shown in Fig. 1, operated on pure oxygen with a background pressure of $1 \times 10^{-4}$ torr $\left(1.33 \times 10^{-2} \mathrm{~Pa}\right)$. The ion source was operated with an anode voltage of $90 \mathrm{~V}$, an anode current of $3.5 \mathrm{~A}$, and a neutralizer current of $100 \mathrm{~mA}$. These end Hall operating parameters produce an average ion energy of $\sim 70 \mathrm{eV}$ (hyperthermal energy) consisting almost exclusively of $\mathrm{O}_{2}{ }^{+}$ions. ${ }^{11}$
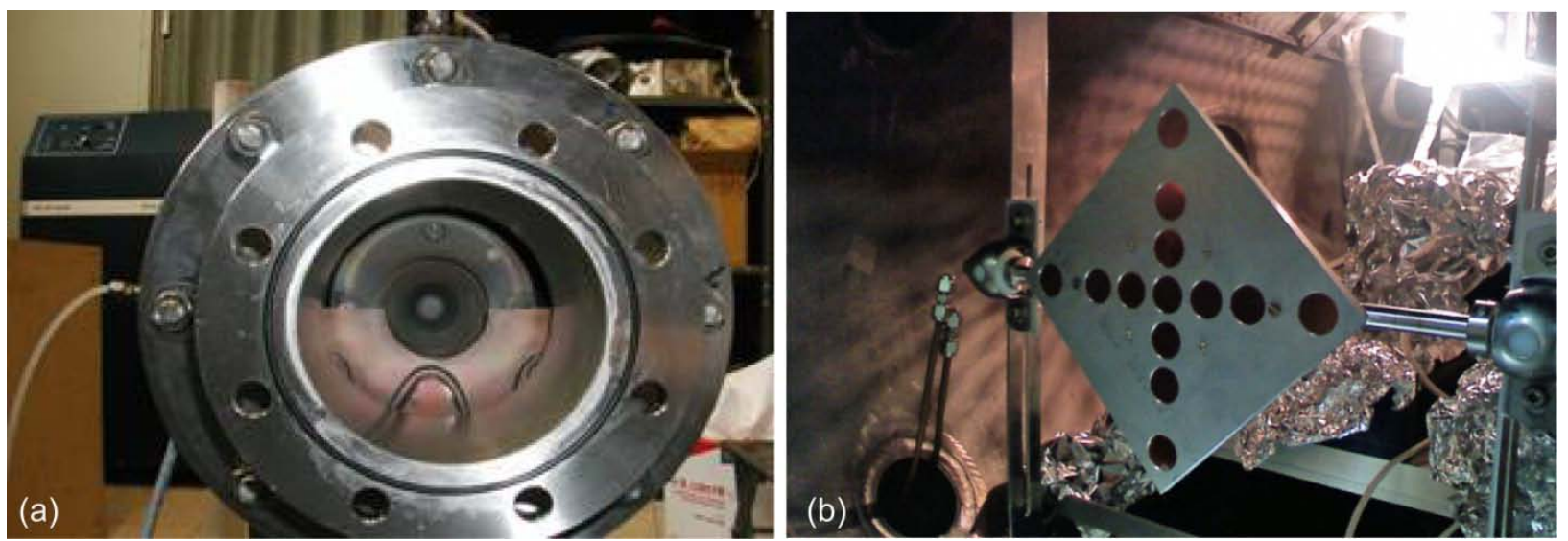

Figure 1. The end Hall oxygen ion source used to texture the samples in this study (a) and the sample holder used (b).

The textured samples were prepared in an end Hall ion source using a sample plate that held multiple samples in the radial direction of the beam to equally distribute the atomic oxygen fluence level. AxFEP and AZ93 thermal control coating samples were exposed for varying duration in hopes of seeing microscopic texture differences on the surface. A total of ten AxFEP samples were exposed for 2, 4, 8 or 16 accumulated hours. Six AZ93 thermal control coatings were exposed for 2,8 or 16 accumulated hours.

Workfunction matching coatings with a thickness of about $100 \mathrm{~nm}$ were sputter deposited onto AZ93 and AxFEP thermal control surfaces. The sputter targets were made of aluminum that had been roughened to allow a slurry of NASA/USGS Lunar Highlands Type (NU-LHT-1D) lunar simulant to be painted on. The slurry-coated targets were oven baked to make an adherent coating on the aluminum substrates for sputter deposition. The coatings were deposited by ion beam sputter deposition using an argon ion beam source to sputter the lunar simulant targets. The resulting coating had a composition similar to the lunar dust simulant, and thus also would have a similar workfunction.

The Ball surface treatments were made by an ion beam surface modification process that operated by supplying a precursor gas(es) into the ion source where a plasma was formed. The positive ions were selectively extracted from the plasma and accelerated out of the ion source providing an ion beam. The ion beam interacted at the substrate surface. The characteristics induced in the substrate surface depends upon the precursor gas(es), gas flow(s), vacuum pressure, ion energy, ion current, distance between the substrate and ion source and several other operational factors. The ion beam treated surface undergoes a chemical change that decreases particle adhesion forces. The ion beam 
vacuum system uses a turbomolecular vacuum pump and an assortment of gases for the ion beam source. The ion beam is water cooled and operates in a vertical position. That is, the materials to be processed are positioned above the ion source. The End Hall ion beam source is a gridless, high ion beam current ( 2 to $8 \mathrm{~A})$ producing source that generates a relatively low ion energy (30 to $300 \mathrm{~V})$ ion beam.

Since the thermal model requires a starting point for the solar absorptance, total reflectance spectra from 250 to $2500 \mathrm{~nm}$ were collected on a Cary 5000 equipped with an integrating sphere. The optical values of the $\alpha$ of the AZ93 samples were used to calibrate the xenon arc lamp for each sample. In many cases, though not all, changes in lamp intensity between the pristine, dusted, and cleaned heating curves were determined by measuring the intensity of the lamp hitting a photo-detector through an orifice plate.

Three types of lunar simulant were used to test the adhesion to the three types of surface treatments. JSC-1AF lunar simulant was used with all three surface treatments. In addition, a 1:1 mixture of JSC-1AF and NU-LHT-1D was used on some of the textured samples, and chromite, an especially dark mineral that has been identified as being on the Moon, was used on some of the workfunction matching coatings. The lunar simulant, with a maximum particle size of $20 \mu \mathrm{m}$, was placed in the Lunar Dust Adhesion Bell Jar (LDAB) and treated with an air plasma to remove organic residue from the grains. It was subsequently dried by heating to $200{ }^{\circ} \mathrm{C}$ in vacuo for 12 to $24 \mathrm{hr}$, and then treated with a hydrogen-helium plasma to chemically reduce their surfaces. The activated dust particles were sieved onto the samples through an electron beam which acted to charge at least some of particles before they hit the thermal control surfaces, to enhance their adhesion since some fraction of the particles on the lunar surface are no doubt ionized by the plasma environment and photo-charging. ${ }^{5}$

The test equipment and procedures have been described in previous reports, and so will not be detailed here. ${ }^{12,9}$ But in summary, the pristine samples are heated in vacuo with a solar simulator and then cooled in a $30 \mathrm{~K}$ cold box. The integrated solar absorptance $(\alpha)$ was determined from the heating curve, and the thermal emittance $(\varepsilon)$ from the cooling curve using Thermal Desktop ${ }^{\circledR}$ modeling software (Cullimore and Ring). Next, samples were sprinkled with activated lunar simulant in vacuo. Once again the samples were heated with a solar simulator and then cooled in a $30 \mathrm{~K}$ cold box.

Since the texture is meant to lower the adhesion of dust to the surface, a procedure was required to assess that. The procedure that was selected involved blowing a calibrated puff of nitrogen gas on the samples. A cylinder with a volume of about $11 \mathrm{~cm}^{3}$ was filled with nitrogen at room temperature and a pressure of $170 \mathrm{kPa}$ absolute (10 psig) for the textured samples. This was increased to $275 \mathrm{kPa}$ absolute $(25 \mathrm{psig})$ for the work function matching coating and Ball's modified surface. A solenoid valve was used to suddenly release the volume into the chamber through two nozzles, one each placed about $1 \mathrm{~cm}$ above the center of each sample. Ideally, this puff of nitrogen would be enough to remove part, but not all of the dust from the samples. Each trial included a pristine sample and a treated sample, one placed under each nozzle. After this puff of nitrogen, again the samples were heated with a solar simulator and then cooled in a $30 \mathrm{~K}$ cold box. By comparing the change in the thermal performance, specifically the ratio $\alpha / \varepsilon$, the relative adhesion of the treated and untreated thermal control surfaces was assessed.

Dust particle counting was carried out on 50 of 641 randomly chosen non-overlapping viewing frames in an optical microscope at a magnification of $100 \times$ and analyzed using ImagePro ${ }^{\circledR}$ software. The procedures are described in detail elsewhere. ${ }^{13}$ Since this value was probably dependant on the amount of dust first sieved onto the sample, the amount of dust initially applied was estimated from the $\alpha$ derived from the heating curve of the dusted sample, using the relation that was derived between dust coverage and the $\alpha$ for that particular dust simulant reported previously. ${ }^{14}$

Since the samples were not uniformly covered with dust at the start of the tests, the total dust remaining after the test is not indicative of coating performance. Probably the best measure of effectiveness of a cleaning technique compares $\alpha / \varepsilon$ of the surface after it has been blown off $(\alpha / \varepsilon)_{b}$ to the $\alpha / \varepsilon$ of the dusted surface $(\alpha / \varepsilon)_{d}$ in terms of the that of the pristine surface $(\alpha / \varepsilon)_{p}$. Equation (1) defines a new term, the dust removal efficiency, $\xi$, as:

$$
\frac{\xi \equiv(\alpha / \varepsilon)_{d}-(\alpha / \varepsilon)_{b}}{(\alpha / \varepsilon)_{d}-(\alpha / \varepsilon)_{p}}
$$

Inspection of this term reveals that if no dust is removed, that is $(\alpha / \varepsilon)_{b}=(\alpha / \varepsilon)_{d}$ then $\xi=0$. If all of the dust has been removed, $(\alpha / \varepsilon)_{b}=(\alpha / \varepsilon)_{p}$ then $\xi=1$. 


\section{Results and Discussion}

\section{A. Textured Surface Samples}

Field emission scanning electron microscopy enables the imaging of the surfaces at high magnification. It is apparent from the photomicrographs in Fig. 2 that the pristine AxFEP surfaces were much smoother than those of the AZ93. After $16 \mathrm{hr}$ of exposure the AgFEP developed surface features around $1 \mu \mathrm{m}$ in size, whereas the AZ93 surface initially had structures near that size. It is difficult to tell from these photographs whether in fact there was any change in the surface roughness due to the ion beam treatment.
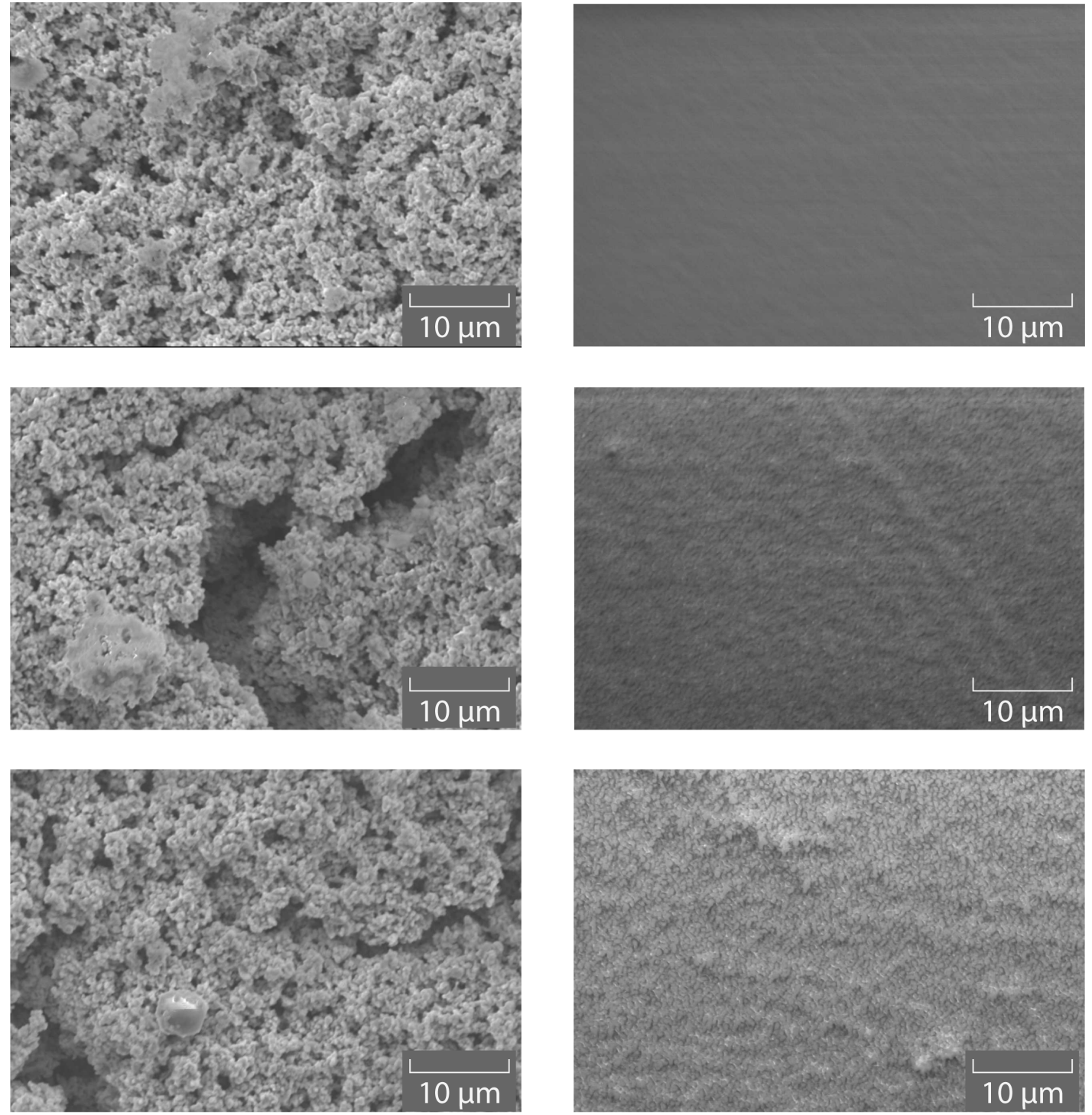

Figure 2. The texture of the AZ93 and AxFEP at 2500x as it develops after 2, 8, and $16 \mathrm{hr}$ exposure to the end Hall oxygen ion beam. 
The optical spectra of the samples from 250 to $2500 \mathrm{~nm}$ before and after texturing were virtually identical. Even the spectra of the AgFEP and AlFEP that were textured for 8 or $16 \mathrm{hr}$, which changed them from specular to diffuse reflectors, were indistinguishable from their pristine counterparts. This indicates that there were no major changes to the samples that would affect their $\alpha$. This was further borne out by the thermal measurements which indicate that the texturing had no significant effect on the $\alpha / \varepsilon$, as seen in Table 1 .

Table 1. The $\alpha / \varepsilon$ of the thermal control surfaces before and after texturing

\begin{tabular}{lccc}
\hline \hline \multicolumn{1}{c}{$\boldsymbol{\alpha} / \boldsymbol{\varepsilon}$} & AZ93 & AlFEP & AgFEP \\
\hline Pristine & $0.166 \pm 0.007$ & $0.22 \pm 0.08$ & ${ }^{\mathrm{a}} 0.09$ \\
Textured & $0.165 \pm 0.008$ & $0.19 \pm 0.02$ & $0.096 \pm 0.002$ \\
\hline
\end{tabular}

${ }^{\mathrm{a}}$ Literature value — no pristine AgFEP samples were used.

Neither the $\alpha$ nor the $\varepsilon$ changed appreciably as the amount of texturing was increased. This is illustrated in Fig. 3 which shows the values of $\alpha$ and $\varepsilon$ as a function of times exposed to the ion beam. It is seen that the values of the textured samples fall within the range of the pristine (time exposed $=0$ ) samples.
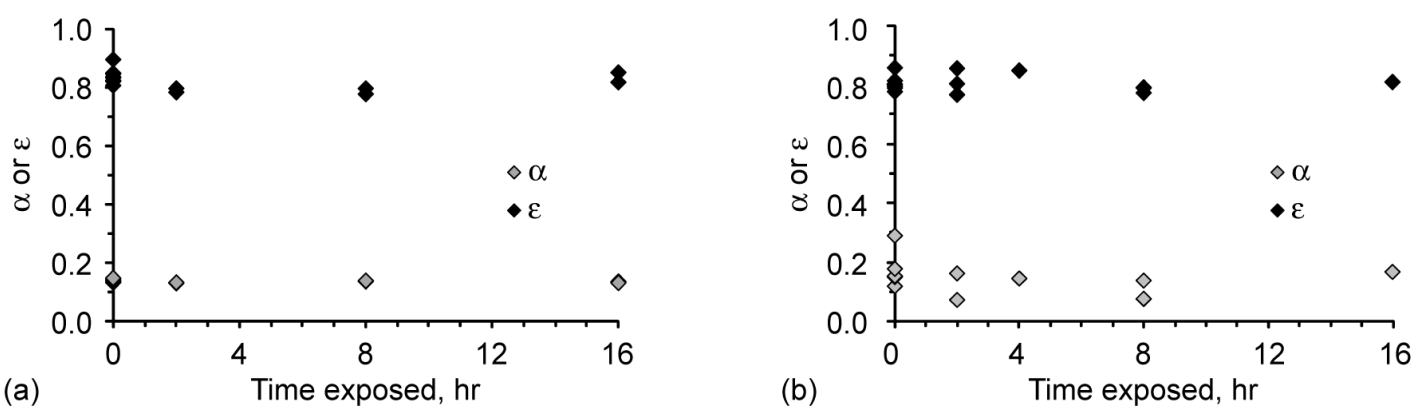

Figure 3. The values of $\alpha$ and $\varepsilon$ derived from the heating and cooling curves in the LDAB as a function of time exposed to the end Hall texturing ion beam for the (a) AZ93 samples and the (b) AgFEP and AIFEP samples. The AgFEP samples are identifiable because their $\alpha$ values are less than 0.1 .

The samples were dusted with a variety of fractional dust coverages in an attempt to determine whether the effectiveness of the texturing was dependent on the amount of dust initially on the surface. That being said, most of the samples were relatively heavily dusted, so that there would be a wide enough difference between the dusted and the pristine thermal optical properties that partial recovery would be detectable. Unfortunately, the protocol for determing the fractional dust coverage cannot be carried out in situ. So the fractional dust coverage before the blowoff tests was calculated from the $\alpha / \varepsilon$ determined from the Thermal Desktop ${ }^{\circledR}$ values that fit the LDAB data. The relationship between the dust coverage and the $\alpha / \varepsilon$ depends upon the lunar simulant used. Further, considerable variation from the least square value is observed. The assumption is that variations from the least square value will be random, so while there will be considerable uncertainty in the value of each data point the data set, when taken as a whole, will have meaning.

The $\alpha / \varepsilon$ of the dusted samples as a function of time exposed to the ion beam for texturing is shown in Fig. 4. Note that the $\alpha / \varepsilon$ is higher for the samples dusted with JSC-1AF, as would be expected from previous studies. The $\alpha / \varepsilon$ of samples of the AZ93 dusted with JSC-1AF all fall within a narrow band because these were all heavily dusted. Though the AZ93 dusted with the JSC-LHT mixture has a much larger range of values, in both cases the range of values of control and textured samples is similar. So the control samples are very much like the textured samples. However, it is noted that in the case of AZ93 dusted with the JSC-LHT mixture that the $\alpha / \varepsilon$ values increase dramatically with the exposier time in the ion beam. It is not suggested that there is a causal relationship here, only that this must be considered as effects of dust cleaning are determined. The AxFEP control samples dusted with the JSC-LHT mixture also span the range of $\alpha / \varepsilon$ values of the textured samples. Once again, the $\alpha / \varepsilon$ values of the AxFEP dusted with the JSC-LHT mixture increase with exposure time in the ion beam, and as before no causal relationship is suggested, but this should be considered when interpreting the dust cleaning results. In the case of the AxFEP samples dusted with JSC-1AF, the three highest $\alpha / \varepsilon$ values occur for textured samples. So the $\alpha / \varepsilon$ values of the control samples do not span the textured samples in this case. 

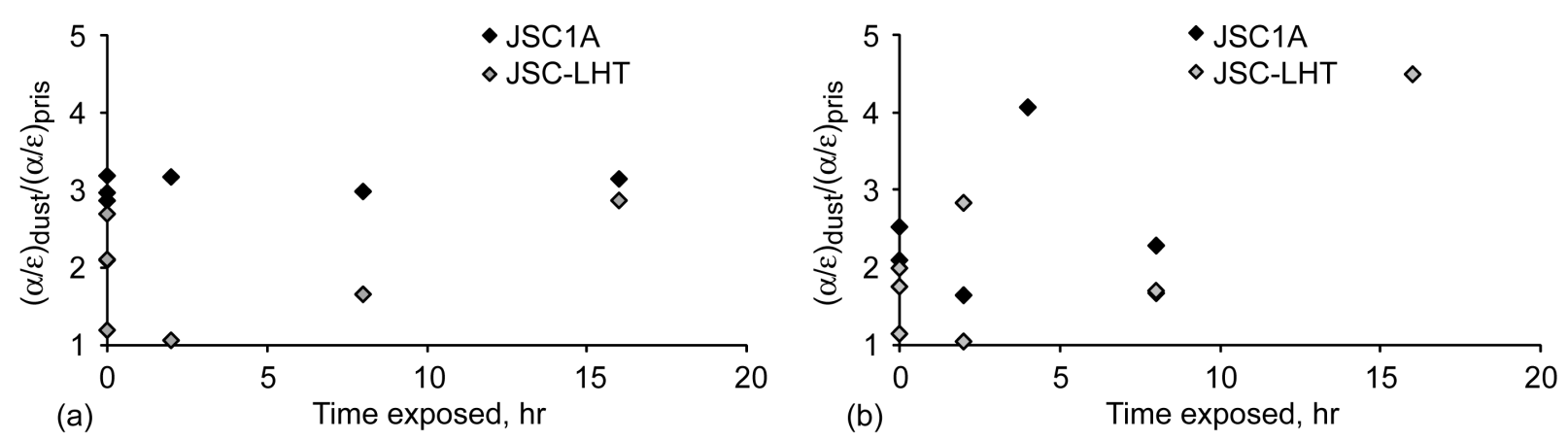

Figure 4. The values of $(\alpha / \varepsilon)_{\text {dust }} /(\alpha / \varepsilon)_{\text {pris }}$ derived from the heating and cooling curves of the samples dusted with JSC-1AF $(\diamond)$ and with the JSC-1AF/NU-LHT-1D mixture $(\diamond)$ in the LDAB as a function of time exposed to the end Hall texturing ion beam for the (a) AZ-93 samples and the (b) AxFEP samples.
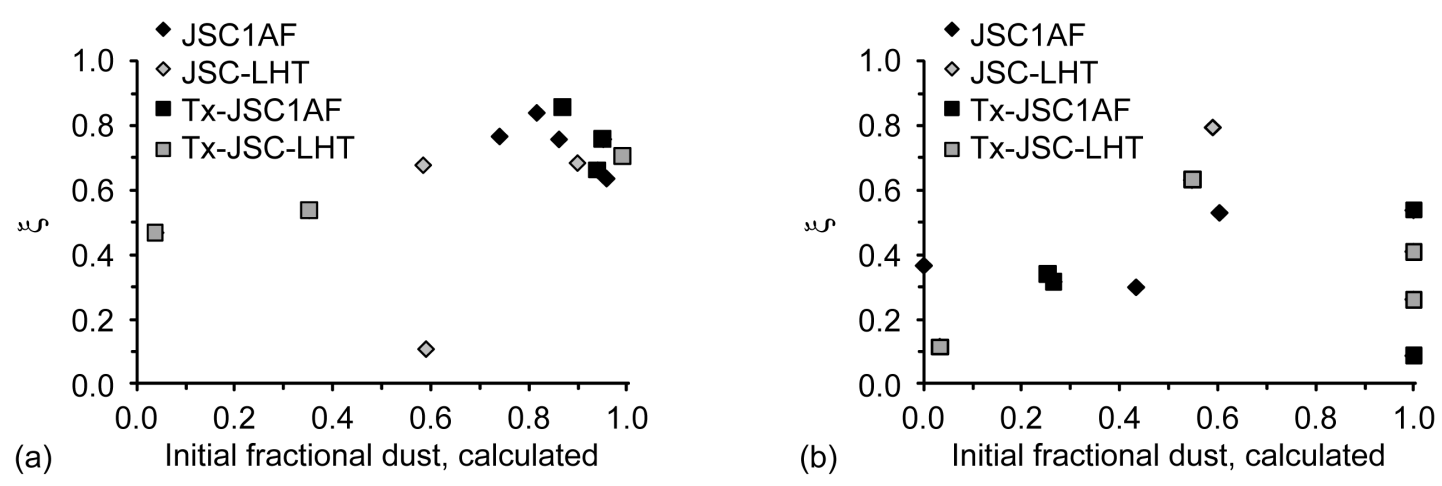

Figure 5. Dust removal efficiency, $\xi$, calculated for pristine and textured (a) AZ93 and (b) AxFEP.

There were some technical problems with the blow-off apparatus which could cause difficulties in quantifying the effectiveness of the surfaces. In the apparatus a tube is filled with nitrogen at a pressure of $170 \mathrm{kPa}$ absolute (10 psig) via a solenoid valve. Then a second solenoid valve is opened causing the volume of nitrogen to flow into the vacuum chamber through a nozzle directed at the sample. This way a standard and repeatable puff of gas to clean the sample is produced. However, during the textured surface experiments there was a small leak in the tube. Although the tube pressurized at $170 \mathrm{kPa}$, when it was released into the vacuum, air was sucked into the chamber as long as the second solenoid valave was open as well. The operator kept the valve open for as similar amount of time as possible for each sample, but there was undoubtably some variation. The result is that these samples were given a larger than intended puff of nitrogen. Since each trial measured a control and a textured sample, random variations should not favor the textured over the control or vice versa. However, comparisons between the textured surfaces and other surface treatments to be tested with the blow-off method are compromised.

The dust removal effectiveness as a function of calculated initial dust coverage is shown in Fig. 5. Since $\xi$ increases with dust coverage, it appears from these plots that it is easier to remove dust from a heavily covered surface than a lightly covered one. This is probably an indication that the adhesive forces of the dust to the surface is greater than the cohesive surfaces among dust particles, since heavily covered surfaces have dust piles of more than monolayer depths. It also appears the the JSC-1AF may be easier to remove than the JSC-LHT mixture, which implies that the JSC-1AF is easier to remove than the NU-LHT-1D. This may be due to particle shape and size distributions. The NU-LHT-1D is somewhat smaller, and appears to have somewhat sharper particles.

Oxygen ion beam texturing appears to have no substantial affect on the $\xi$ of AZ93. In retrospect, this is not particularly surprising given that the paint initially has texture on the same order as that generated by the ion beam. The ion beam texturing also did not appear to reduce the adhesion of either the JSC-1AF or the JSC-LHT to AxFEP. It is noted that four of the textured samples had particularly heavy dust layers applied to them as the calculated initial dust fraction was greater than 1.0. Perhaps much of the dust was removed but after the blow off a considerable amount of dust still remained, so the $\xi$ value was still low. But even if those data are ignored, the textured samples had no higher $\xi$ than the pristine samples. Oxygen beam texturing was not an effective strategy to lower the adhesion for either thermal control surface. 


\section{B. Work Function Matching Coatings}

The optical spectra of the samples before and after applying the work function matching coating were virtually identical. There was no visual evidence of the coating, and Table 2 shows there was no substantial difference in the integrated $\alpha / \varepsilon$ of the AZ93, but there may have been as much as a 10 percent increase in the AxFEP samples.

Table 2. The $\alpha / \varepsilon$ of the thermal control surfaces before and after applying the work function matching coating

\begin{tabular}{cccc}
\hline \hline$\alpha / \boldsymbol{\varepsilon}$ & AZ93 & AlFEP & AgFEP \\
\hline Pristine & $0.199 \pm 0.004$ & $0.20 \pm 0.01$ & ${ }^{\mathrm{a}} 0.08$ \\
Coated & $0.193 \pm 0.005$ & $0.22 \pm 0.06$ & ${ }^{\mathrm{b}} 0.10$ \\
\hline \hline
\end{tabular}

${ }^{\text {a }}$ Single sample

${ }^{\mathrm{b}}$ Two samples

As would be expected from the similar spectra, the $\alpha, \varepsilon$, and $\alpha / \varepsilon$ of the thermal control coatings did not change appreciably upon the addition of the work function matching coating at either thickness. This is shown in Fig. 6. It can be seen that the values of the coated samples fall within the range of the pristine (time exposed $=0$ ) samples.

These samples were dusted also with a variety of fractional dust coverages in an attempt to determine whether the effectiveness of the texturing was dependant on the amount of dust initially on the surface. As before the fractional dust coverage before the blow-off tests was calculated from the $\alpha / \varepsilon$ determined from previous experiments. The relationship between the dust coverage and the $\alpha / \varepsilon$ depends upon the lunar simulant and the thermal control surface used. Further, considerable variation from the least square value is observed. The assumption is that variations from the least square value will be random, so while there will be considerable uncertainty in the value of each data point the data set, when taken as a whole, will have meaning. The results are shown in Fig. 7.

The dust removal effectiveness as a function of calculated initial dust coverage is shown in Fig. 8. The $\xi$ for the workfunction matching coating on AZ93 appear to unchanged from the uncoated surfaces (Fig. 3(a)) over a wide span of dust coverage. It is noted that the JSC-1 AF appears to be easier to remove from the AZ93 surfaces than the chromite. Figure 3(b) shows that workfunction matching coatings did have a large effect on the ability to clean the dust off using a nitrogen puff. Under these test conditions less than 10 percent of the dust was removed from the uncoated surfaces, but 20 to 40 percent of chromite was removed from the coated surfaces, and 50 to 80 percent of the JSC-1AF. These results suggest that the workfunction matching coatings, combined with a puff of gas, could be an effective way to remove dust from metal-backed FEP thermal control surfaces on the lunar surface.
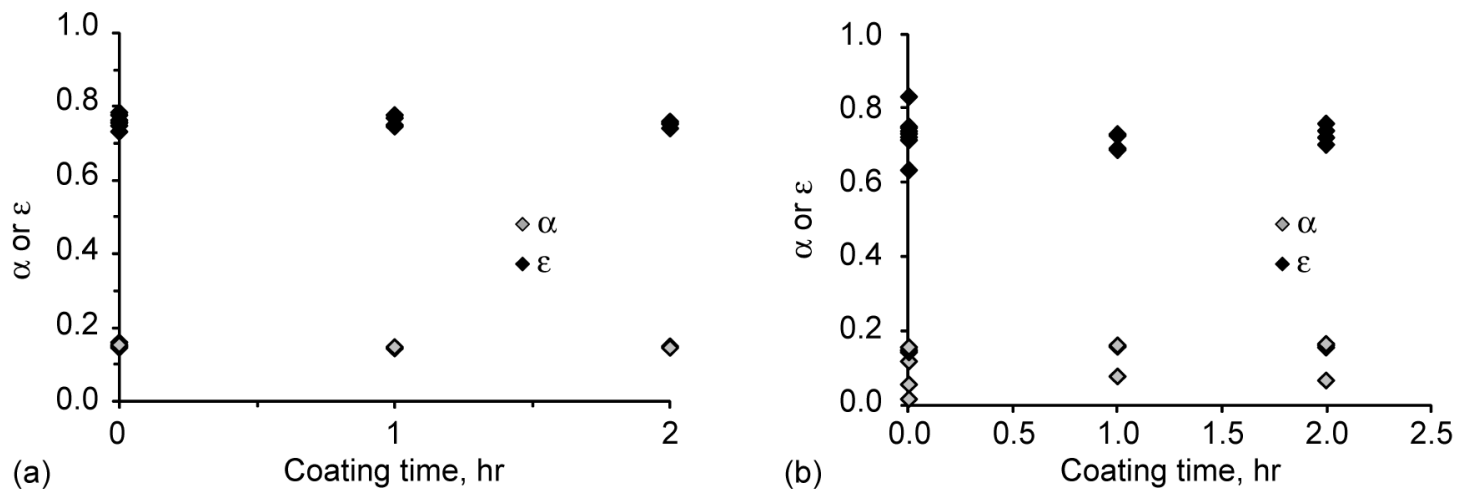

Figure 6. The values of $\alpha$ and $\varepsilon$ derived from the heating and cooling curves in the LDAB as a function of time exposed to the sputter deposition for the (a) AZ93 samples and the (b) AxFEP samples. Samples in Figure 6(b) with $\alpha<0.1$ are AgFEP, the rest are AlFEP. 

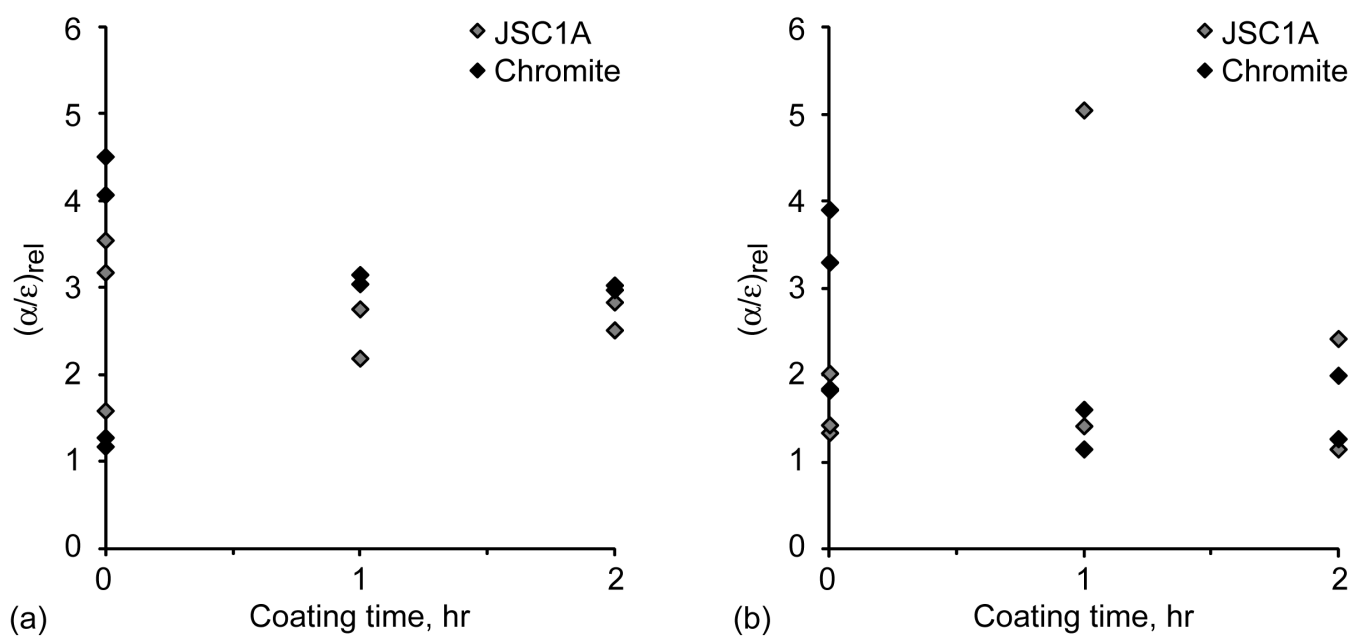

Figure 7. The values of $(\alpha / \varepsilon)_{\text {rel }}$ derived from the heating and cooling curves of the samples dusted with chromite $(\diamond)$ and with JSC-1AF $(\diamond)$ in the LDAB as a function of deposition time of sputter deposited work function matching coatings for the (a) AZ-93 samples and the (b) AxFEP samples.
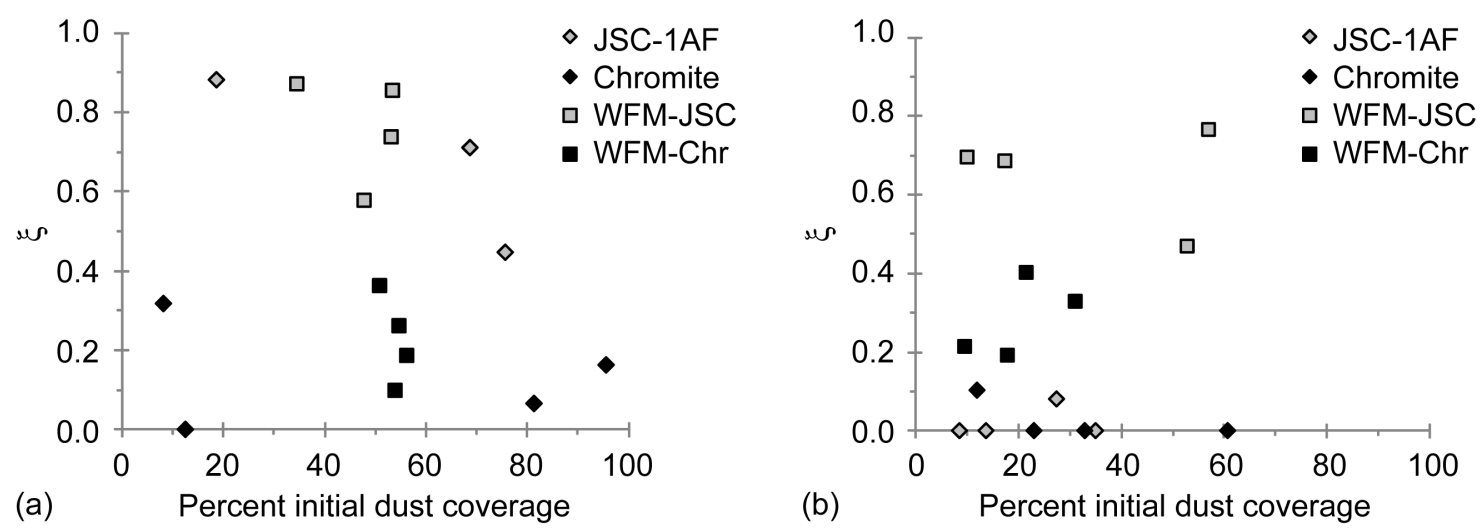

Figure 8. Dust removal efficiency, $\xi$, calculated for pristine and workfunction matching coated (a) AZ93 and (b) AxFEP. 


\section{Ball Aerospace Surface Treatment}

The average optical spectra of pristine and treated AZ93 and AgFEP are shown in Fig. 9. There was almost no spread in the spectra of the AgFEP across the entire spectrum, but there was a little spread in the AZ93 spectra, particularly in the region above about $1800 \mathrm{~nm}$. The differences between the pristine and treated spectra were small but when convoluted with the AM0 solar spectrum gave slightly different values of $\alpha$.
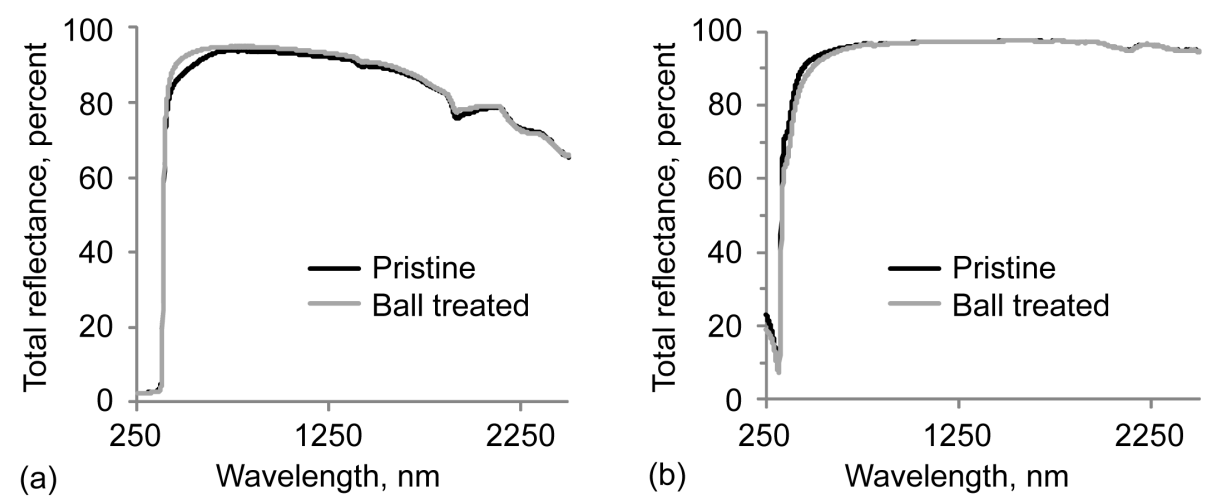

Figure 9. Total reflectance spectra of pristine and Ball treated samples of (a) AZ93 and (b) AgFEP used in this study.

Each individual spectrum was convoluted with the ASTM air mass zero (AM0) spectrum in order to determine the total integrated solar absorptance $(\alpha)$. Those values are shown in Table 3. The $\alpha$ of AgFEP increases about 13 percent because of the surface treatment, while the $\alpha$ of the AZ93 decreases about 6 percent (though there is about 3 percent spread in the data). The $\alpha$ as well as the $\alpha / \varepsilon$ that was determined by thermal analysis of the heating and cooling curves are also shown in Table 3 for comparison.

Table 3. Integrated total solar absorptance $(\alpha)$ determined spectrally and thermally for undusted pristine and Ball treated thermal control surfaces, as well as the thermally determined $\alpha / \varepsilon$.

\begin{tabular}{lccc}
\hline \hline \multicolumn{1}{c}{ Material } & Optical $\alpha$ & Thermal $\alpha$ & Thermal $\alpha / \varepsilon$ \\
\hline AgFEP & $0.0953 \pm 0.0004$ & $0.077 \pm 0.003$ & $0.097 \pm 0.005$ \\
Ball AgFEP & $0.1074 \pm 0.0009$ & $0.080 \pm 0.001$ & $0.102 \pm 0.007$ \\
AZ93 & $0.171 \pm 0.006$ & $0.168 \pm 0.007$ & $0.212 \pm 0.012$ \\
Ball AZ93 & $0.162 \pm 0.004$ & $0.161 \pm 0.002$ & $0.205 \pm 0.008$ \\
\hline \hline
\end{tabular}

The dust removal effectiveness as a function of calculated initial dust coverage is shown in Fig. 10. The $\xi$ for the Ball surface treatment on AZ93 appears to be unchanged from the untreated surfaces (Fig. 2(a)) with an average of 0.74 for the pristine and 0.75 for the surface treated, well within the standard deviation of about 0.2 . Figure $10(\mathrm{~b})$ shows that the Ball surface treatment did improve the ability to clean the dust off of AgFEP using a nitrogen puff. Under these test conditions the average $\xi$ was 0.23 for the pristine but 0.67 for the treated. These results show that the Ball surface treatment lowers the adhesion of the AgFEP surface significantly and should be considered for further studies.

Four of the surface treated AZ93 samples and four of the surface treated AgFEP samples were also included in a separate study to test the effectiveness of brushing JSC-1 AF lunar simulant dust in the LDAB. Two of each were tested using a fiberglass bristle fingerprint brush (Zephyr) and two of each with a nylon bristle fan brush (Escoda). Both brushes were found to be effective at restoring the $\alpha / \varepsilon$ to a level of $\xi=0.80$ after 20 strokes and $\xi=0.90$ after 200 strokes. Although the samples size was small, the Ball Aerospace treated samples did not appear to perform either better or worse than the untreated samples. 

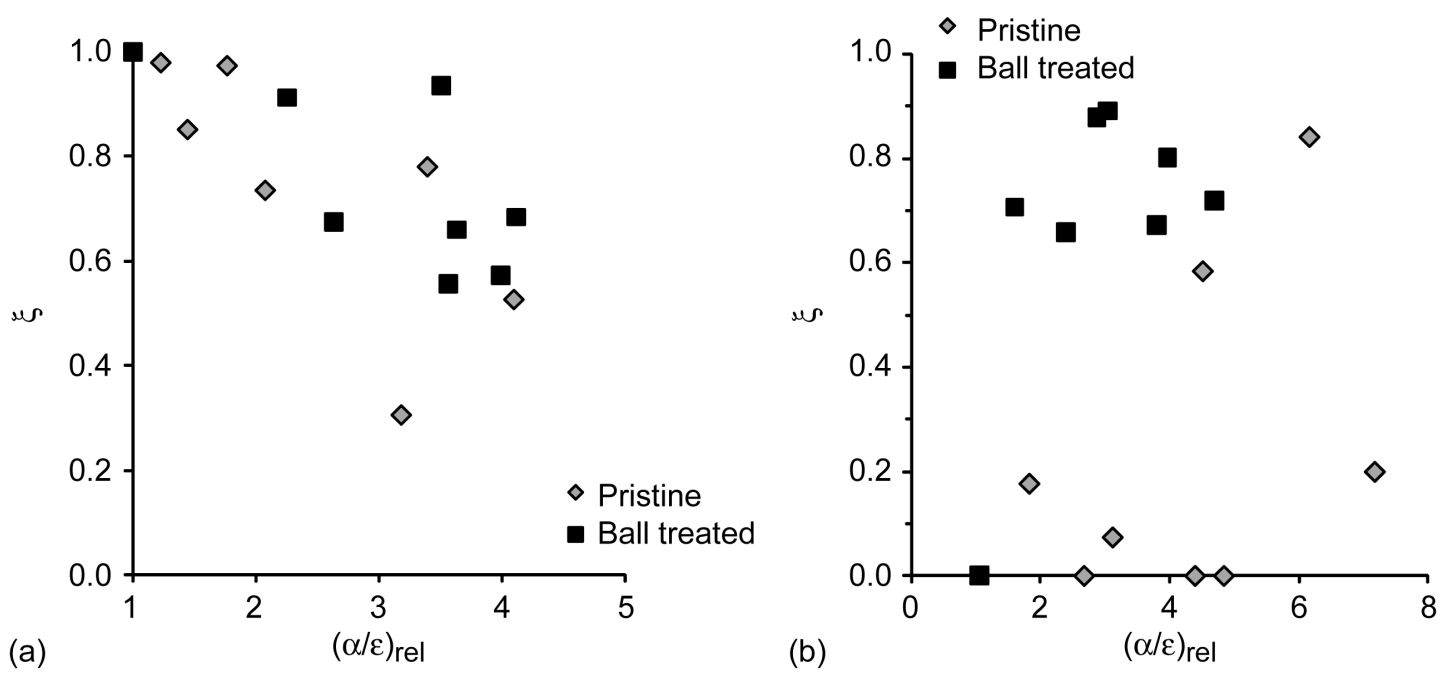

Figure 10. Dust removal efficiency, $\xi$, calculated for pristine and Ball surface treated (a) AZ93 and (b) AgFEP.

\section{Conclusions}

Three surface treatments were evaluated for their ability to lower the adhesion between lunar simulant dust and AZ93, AlFEP, and AgFEP under simulated lunar conditions. The first treatment was oxygen ion beam texturing of the thermal control surface. This produced surface features about $1 \mu \mathrm{m}$ is size without significantly changing the surface chemistry. The second treatment was the deposition of a $100 \mathrm{~nm}$ thick layer coating of the same composition as the lunar simulant with presumably a similar work function, to minimize charge transfer between the dust and the surface. The third treatment was a Ball proprietary ion beam process that results in a modified surface with altered surface chemistry.

The test was to determine whether more dust was removed from the treated thermal control surface than the pristine surface when subjected to a pulse of nitrogen gas through a nozzle over the samples. Due to slight experimental variations, a direct comparison among the three treatments from these data is not advisable. It was noted it was easier to remove a large fraction of a heavy dust layer than a large fraction of a sparse dust layer by removing the dust using a gas jet. Although the study was not designed to test the amount of gas to remove dust from the surface, the results suggest that this may be an effective method to reduce the thickness dust layer. A viable strategy might be to use a gas jet to remove enough of the dust to return the thermal control surface to viability, even if not to restore to pristine condition.

None of the three treatments significantly improved the removal of dust from AZ93 white paint. The pristine paint has surface features that are on the order of $1 \mu \mathrm{m}$, so etching the surface with oxygen produced little change. AZ93 uses a metal oxide pigment in a silicate binder, so the surface chemistry is already similar to that of the mineral dust. Although the exact nature of the Ball treatment is proprietary, it also did not affect the adhesion between dust and the paint.

The oxygen ion beam texturing of the AxFEP samples also proved ineffective for lowering the adhesion of dust. But both the work 3 function matching coating and the Ball surface treatment appear to substantially decrease the adhesion of lunar simulant. In the case of the work function matching coating, every coated sample had more dust removed than any of the untreated samples. It was also noted that all of the samples dusted with JSC-1AF simulant had higher $\xi$ values than any of the chromite dusted samples. However, the same coverage of chromite, being a darker simulant, more strongly degrades the $\alpha$ of the thermal control surface. Although there were some pristine samples with as high a $\xi$ as the Ball treated samples, the average value of $\xi$ was much higher for the Ball treated. It is recommended that both of these surface treatments be explored further as dust resistant coatings for AxFEP thermal control surfaces. 


\section{Acknowledgements}

This work is the result of a great deal of effort by a sizable team of dedicated professionals from many organizations, all of whom the author would like to thank. E.A. Sechkar (Arctic Slope Regional Corporation) and F.P. Lam (Jacobs Sverdrup) provided engineering and technical support of the LDAB facility. S.R. Panko (Arctic Slope Regional Corporation) provided both electrical engineering and test engineering support for the LDAB. The thermal modeling was a team effort involving several people from the NASA Glenn Research Center (GRC) Thermal Systems Branch. D.A. Jaworske (GRC) oversaw the fabrication of the test samples and acted as a general resource on the behavior of thermal control coatings. Technical, programmatic, and financial support was provided by the Exploration Technology Development Program's Dust Mitigation Project (M.J. Hyatt, GRC) and Advanced Thermal Control Project (R.A. Stephan, NASA Johnson Space Center).

\section{References}

${ }^{1}$ J.R. Gaier, "The Effect of Lunar Dust on EVA Systems During the Apollo Missions," NASA Technical Memorandum, NASA/TM-2005-213610/REV1, (2005).

${ }^{2}$ J.R. Gaier and D.A. Jaworske, "Lunar Dust on Heat Rejection System Surfaces: Problems and Prospects," Space Technologies and Applications International Forum 2007. (Also, NASA/TM-2007-214814)

${ }^{3}$ J.R. Gaier, "Effect of Lunar Simulant Type on the Absorptivity and Emissivity of Dusted Thermal Control Surfaces in a Simulated Lunar Environment," International Conference on Environmental Systems 2010, AIAA-2010-6111. Also NASA/TM-2010-216786.

${ }^{4}$ P.S. Greenberg, D-R Chen, and S.A. Smith, "Aerosol Measurements of the Fine and Ultrafine Particle Content of the Lunar Regolith,” NASA/TM-2007-214956.

${ }^{5}$ L.A. Taylor, Y. Liu, and A. Zhang, "Shape and Size Relationship of Several Lunar Dusts: Preliminary Results," $40^{\text {th }}$ Lunar and Planetary Science Conference (2009) 2106.pdf.

${ }^{6}$ J.J. Grossman, et al., "Microchemical, Microphysical and Adhesive Properties of Lunar Material," Proceedings of the Apollo 11 Lunar Science Conference, Vol. 3, pp. 2171-81.

${ }^{7}$ W.M. Farrell, et al., "Complex Electric Fields Near the Lunar Terminator: The Near-Surface Wake and Accelerated Dust," Geophys. Res. Let., Vol. 34, L14201, doi: 10.1029/2007GL029312 (2007).

${ }^{8}$ Z. Sternovsky, et al., "Contact Charging of Lunar and Martian Dust Simulants," J. Geophys. Res. Vol. 107, E11, 5105, doi: 10.1029/2002JE001897 (2002).

${ }^{9}$ M.S. Crowder, et al., "Reducing Particle Adhesion by Material Surface Engineering: Proc of SPIE, 77940G-1 (2010).

${ }^{10}$ M.S. Crowder, NASA Contract NNM09AA11C, "Contamination Resistant Surfaces for Lunar Dust Mitigation".

${ }^{11}$ J.R. Kahn, and R.S. Robinson, NASA contract report, Front Range Research, Fort Collins, CO, Sept. $28,1989$.

${ }^{12}$ J.R. Gaier and E.A. Sechkar, "Lunar Simulation in the Lunar Dust Adhesion Bell Jar," Proceedings of the 45th AIAA Aerospace Sciences Meeting (2007).

${ }^{13}$ J.R. Gaier, J. Siamidis, and E.M.G. Larkin, "Extraction of Thermal Performance Values from Samples in the Lunar Dust Adhesion Bell Jar," $25^{\text {th }}$ Space Simulation Conference, NASA/CP-2008-214164.

${ }^{14}$ J. R. Gaier, "Measurement of the Solar Absorptivity and Thermal Emissivity of Lunar Simulants," International Conference on Environmental Systems 2010, AIAA-2010-6025. Also NASA/TM-2010-216788. 